\title{
A reversal in the direction of detection asymmetry: Effects of spatial density, spatial regularity, and retinal eccentricity
}

\author{
CRISTINA MEINECKE \\ Ludwig-Maximilians-Universität, Munich, Germany \\ RUTH KIMCHI \\ University of Haifa, Haifa, Israel \\ and \\ CHRISTINA GRANDEGGER \\ Max-Planck-Institut für Psychologische Forschung, Munich, Germany
}

\begin{abstract}
We analyzed detection asymmetry, using open and closed squares as targetand context elements. It was found that varying density, regularity, and target eccentricity not only can modulate the amount of asymmetry, but also can produce a reversal in the direction of the asymmetry. The results suggest that the different stimuli are processed with different grains of analysis, consisting of in some cases, single elements and their properties and, in other cases, larger units of the stimulus array and more global properties. A detection task that can utilize the properties that are available with a given grain of analysis would show an advantage over a task for which the given grain of analysis is not as favorable, resulting in detection asymmetry in one direction or another.
\end{abstract}

It is often easier to detect an element A embedded in a context of elements B than vice versa. This detection asymmetry has been found in visual search tasks and in texture discrimination tasks (e.g., Beck, 1973, 1982; Carrasco, McLean, Katz, \& Frieder, 1998; Gurnsey \& Browse, 1987, 1989; Julesz \& Bergen, 1983; Meinecke, 1989; Rubenstein \& Sagi, 1996; A. M. Treisman \& Gormican, 1988; A. M. Treisman \& Souther, 1985; Williams, 1992).

Detection asymmetries are observed with stimuli that are composed of several single elements, some of which are defined as targets. They are especially pronounced when the number of context elements is clearly larger than the number of elements that form the target. In visual search tasks (e.g., A. M. Treisman \& Souther, 1985), asymmetries with a specific stimulus material increased markedly with increasing display size (i.e., with an increasing number of context elements).

The magnitude of asymmetries is rather variable, depending on the pair of stimuli used to construct the target and the context, respectively. For example, Gurnsey and

\footnotetext{
We thank Jeremy M. Wolfe, Marisa Carrasco, and an anonymous reviewer for useful comments on earlier versions of this article. Correspondence concerning this article should be addressed to C. Meinecke, Institut für Psychologie, Universität Erlangen-Nürnberg, Kochstr. 4, D-91054Erlangen, Germany (e-mail: meinecke@ rzmail.uni-erlangen.de) or to R. Kimchi, Department of Psychology, University of Haifa, Haifa 31905, Israel (e-mail: rkimchi@ @research.haifa.ac.il).
}

Browse (1987) and A. M. Treisman and Gormican (1988) tested a large number of different pairs that produced asymmetries in all magnitudes, from zero to very pronounced.

How are asymmetries explained? The most prevailing account, proposed by A. M. Treisman (e.g., A. M. Treisman \& Gormican, 1988), attributes detection asymmetries to specific properties in the target element. Thus, a target element may have a specific property that is not present in the context elements (e.g., terminators), is present to a lesser quantity in the context elements (e.g., length), or deviates from a "standard" or a prototype present in the context elements (e.g., tilt, curvature). A. M. Treisman (A. M. Treisman \& Gormican, 1988; A. M. Treisman \& Souther, 1985 ) suggested that, in all these cases, the context elements elicit less activity than does the target (because the target element has something in addition to what is present in the context element) and the detection of a highactivity element among low-activity elements is more efficient than vice versa. We term this hypothesis the moreis-better hypothesis. The assumptions underlying this hypothesis are (1) that search for a target is accomplished by a detection of a unique element and (2) that asymmetry arises when the context elements generate less activity than the target. This hypothesis is supported by findings that indicate detection asymmetry when the target element is larger than the context elements (see Gurnsey \& Browse, 1989), is longer (see A. M. Treisman \& Gormican, 1988), or has 
an additional subelement, such as the small line in the letter Q (see A. M. Treisman \& Souther, 1985).

The more-is-better hypothesis, however, does not seem to adequately explain all cases of detection asymmetry. For example, a tilted target among vertical distractors is more easily detected than a vertical target among tilted distractors (e.g., Carrasco \& Frieder, 1997; A. M. Treisman \& Gormican, 1988). Yet, as was pointed out by Carrasco and Frieder, the assumption that vertical lines would generate less activity than diagonal ones, required by the more-isbetter hypothesis to account for the orientation asymmetry, is not supported by physiological and psychophysical data (e.g., the oblique effect; Appelle, 1972).

An alternative to the more-is-better hypothesis emphasizes properties of the context and detection of discontinuity or irregularity, rather then finding the unique element in the display (e.g., Carrasco \& Frieder, 1997; Carrasco et al., 1998; Sagi \& Julesz, 1987). We term this alternative hypothesis the deviation hypothesis. The assumptions underlying the deviation hypothesis are (1) that search for a target is accomplished by detection of discontinuity (i.e., a deviation from continuity or regularity) in the display and (2) that asymmetry arises when the context elements are processed more efficiently than the target, so that they can be easily perceived and grouped to allow the detection of the discontinuity of the target element. Thus, for example, Carrasco and Frieder suggested that orientation asymmetries may be due to a more efficient processing of the vertical than of the tilted lines, owing to the low threshold needed to detect vertical lines, so that the discontinuity of a tilted line among vertical ones is detected more easily than the discontinuity of a vertical line among tilted ones. The deviation hypothesis is supported by findings that show detection asymmetry when the context best allows the detection of a deviation, a discontinuity. That is, when the context is spatially regular (see Gurnsey \& Browse, 1989), is optimal for specialized cells in our visual system (e.g., vertically and horizontally oriented filters, see Foster \& Ward, 1991; spatial frequency filters, Carrasco et al., 1998), or is well known (see the familiarity effect; e.g., Meinecke, 1995, 1997; Wang, Cavanagh, \& Green, 1994).

One problem with these hypotheses is that they do not take into account the possible effects of properties of the visual system, such as resolution as a function of eccentricity, and the spatial properties of the stimulus display, such as density and regularity, on detection asymmetry, although both types of properties have been found to affect detection performance. Thus, detection performance can take very different courses as a function of retinal eccentricity of target projection. A decrease in detection performance with increasing eccentricity has been found in visual search studies (e.g., Carrasco \& Frieder, 1997; Carrasco et al., 1998). In some studies with more texturelike stimuli, it has been found that detection performance increases with increasing eccentricity and then decreases (central performance drop, CPD; e.g., Gurnsey, Pearson, \& Day, 1996; Joffe \& Scialfa, 1995; Kehrer, 1987, 1989;
Meinecke \& Kehrer, 1994; Yeshurun \& Carrasco, 1998). This latter finding suggests that, in some cases, detection performance can actually benefit from a certain decrease in resolution (e.g., Gurnsey et al., 1996; Kehrer, 1987, 1989; Meinecke, 1989). Effects of spatial properties of the stimulus array on detection performance also have been reported in the literature. Increased density has been found to improve detection performance in some cases (e.g., Nothdurft, 1985; Sagi \& Julesz, 1987), but not in others (e.g., Bacon \& Egeth, 1991; Kimchi \& Navon, 2000), and spatial irregularity impairs detection (Gurnsey \& Browse, 1989; Kehrer, 1987; Nothdurft, 1990, 1991).

Detection asymmetries can also be affected by target eccentricity (Carrasco et al., 1998; Meinecke, 1989; Poirier \& Gurnsey, 1998; Rubenstein \& Sagi, 1996). For example, Meinecke (1989) found that the detection of an O embedded in Hs was more efficient than the detection of an $\mathrm{H}$ embedded in Os only when the target was projected outside the foveal area of the retina. Carrasco et al. found that a tilted line embedded in vertical lines yielded a more efficient detection than did a vertical line embedded in tilted lines and that this asymmetry increased with increasing retinal eccentricity of the target. That is, the asymmetry was coupled with a specific processing of the stimulus that depended on the specific properties of the visual system as a function of eccentricity.

Likewise, the spatial arrangement of the elements in the display also seems to have an effect on detection asymmetry. For example, Meinecke (1989) showed that increasing the distance between the elements decreased the advantage of angular context over round context in the peripheral areas, and Rubenstein and Sagi (1996) showed that the advantage of open-target-closed-context elements over closed-target-open-contextelements observed in visual search tasks (e.g., A. M. Treisman \& Souther, 1985) did not surface in peripheral areas when the elements were arranged in a texture matrix.

These findings, showing that specific stimulus properties that are more effective for target detection under certain eccentricity and display density may not be as effective under different eccentricity and density, could be regarded just as evidence for limits to generalizations about feature detectability. But they might also serve to enlighten us about factors that determine detection performance. Therefore, in the present study, we attempted to better delineate the conditions under which detection asymmetry is manifested.

We hypothesize that the grain of analy sis varies as a function of retinal eccentricity (Meinecke, 1989) and stimulus density, so that properties of single elements are likely to be best processed in the foveal area and under low density, whereas more global properties that are computed over larger sampling units may not be hindered or may even benefit from high stimulus density and increased eccentricities. Consequently, a task that can be performed on the basis of information that is best supported by a given grain of analysis is likely to show an advantage over a task for which the given grain of analysis is not favorable. This may 
result in detection asymmetry and, perhaps even in a reversal of asymmetry.

From this point of view, the two hypotheses mentioned above may not be incompatible. The more-is-better hypothesis, which focuses on the analysis of the properties of single elements, would account for asymmetry in the foveal area, where acuity is high enough to analyze details, and in sparse displays that are less likely to produce global properties. The deviation hypothesis, which focuses on detection of irregularities or discontinuities, would account for asymmetry in more peripheral areas or in dense displays in which larger sampling units allow a spatial integration of information.

As stimulus material, we chose open and closed elements that already had been found to produce an asymmetry in visual search tasks: It is easier to find an open element embedded in closed elements than vice versa (e.g., A. M. Treisman \& Souther, 1985; Williams, 1992). The more-is-better hypothesis (A. M. Treisman \& Gormican, 1988) attributes this asymmetrical performance to the ease of detecting the element with a unique feature in the display - the element with terminators (the open element has terminators, whereas the closed elements do not). The deviation hypothesis, on the other hand, attributes this asymmetry to the more efficient processing of the closed elements than of the open ones, which facilitates the perception of the closed background elements to reveal the discontinuity of the open element. Thus, both explanations predict better performance in the case in which closed elements form the context. This study explores the effects of the spatial properties of the stimulus and target eccentricity on detection performance.

In Experiment 1, we compared detection performance with these stimuli while varying the spatial properties of the stimulus (dense regular display vs. sparse and irregular display) and the eccentricity of the target. With our sparse irregular displays, we replicated the asymmetry reported in previous studies and predicted by the two aforementioned hypotheses but found a reversal of the asymmetry with the dense regular display. In the next experiments, we then attempted to assess the relative contribution of density and regularity to the reversal effect and to examine the effect of eccentricity under the different stimulus conditions.

\section{EXPERIMENT 1 \\ Dense Regular Stimuli Versus Sparse Irregular Stimuli}

In this experiment, we compared detection performance with highly dense regular stimuli with detection performance with sparse irregular stimuli (similar to the ones typically used in visual search tasks) at different retinal eccentricities of the target.

\section{Method}

Participants. Eight participants (5 women and 3 men) were paid to participate in the experiment. They were $21-35$ years old. All the participants in this experiment and in all the other experiments had normal or fully corrected visual acuity (visual acuity test with a Rodenstock R12 Vision Tester, test stimuli No. 112).

Apparatus. The experiment was controlled by an Atari Mega STE computer, and the stimuli were presented on an Atari SM 144 screen. The participant sat at a viewing distance of $40 \mathrm{~cm}$, with the direction of gaze inclined slightly downward, and his or her head was resting on a head- and chin-rest. The participant responded to the stimuli by pressing one of the two mouse keys.

Stimuli. The stimuli are depicted in Figure 1. The elements were open or closed squares. ${ }^{1}$ They were constructed in an $8 \times 8$ pixel matrix $\left(3.04 \mathrm{~mm}\right.$ in the vertical and horizontal directions; $0.44^{\circ} \times$ $0.44^{\circ}$ of visual angle). The elements were black $\left(0.05 \mathrm{~cd} / \mathrm{m}^{2}\right)$ and the screen background was white/gray $\left(37.5 \mathrm{~cd} / \mathrm{m}^{2}\right)$.

In the dense regular conditions, the stimuli consisted of $35 \times 7$ regularly spaced elements, centered on the screen; the distance between the single elements was $1.14 \mathrm{~mm}\left(0.16^{\circ}\right)$ in the horizontal and vertical directions. The stimulus subtended $14.52 \mathrm{~cm}\left(19.95^{\circ}\right)$ in the horizontal direction and $2.81 \mathrm{~cm}\left(4.02^{\circ}\right)$ in the vertical direction. The target consisted of three elements arranged vertically in a row (vertical extension: $11.40 \mathrm{~mm}, 1.63^{\circ}$ ). In the context of open squares, the target consisted of closed squares, and vice versa. The vertical position of the target was always centered in the stimulus. The horizontal position of the target varied: The target could appear at every 2 nd position of the central 23 positions Thus, the target could appear at 13

\section{Dense regular}

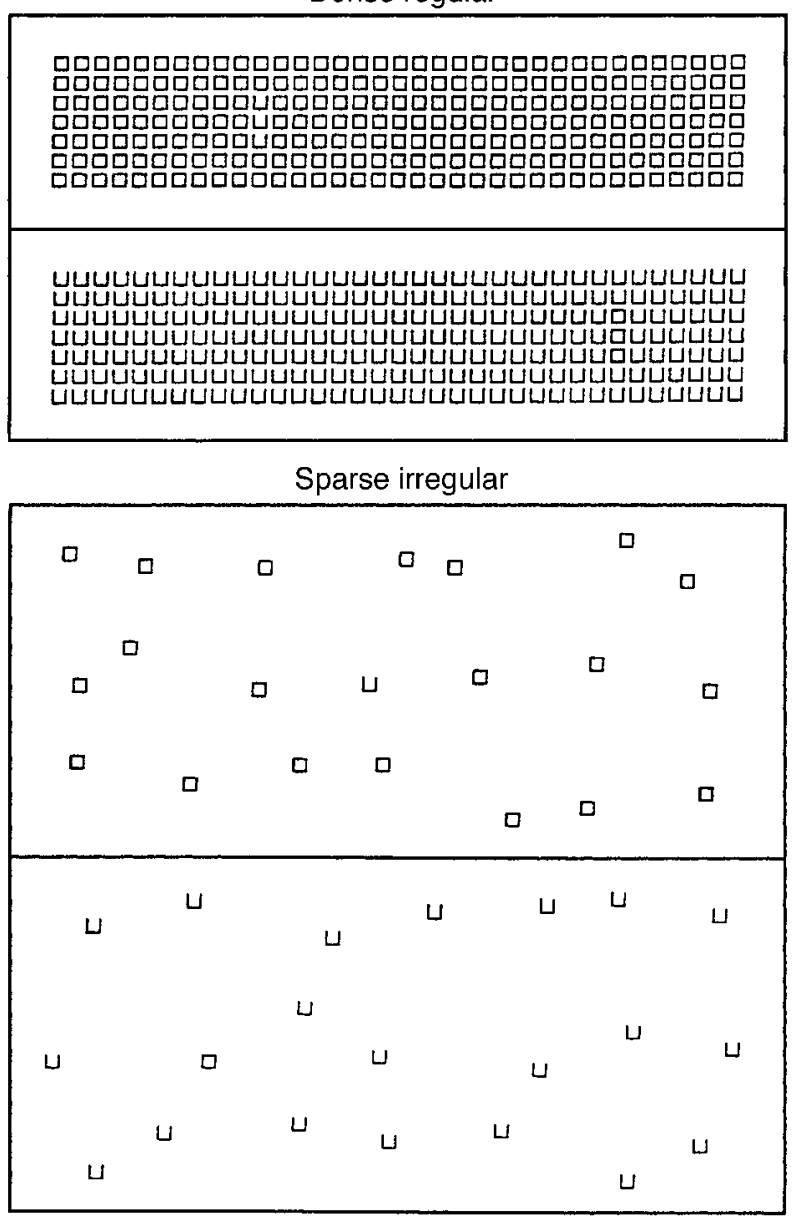

Figure 1. Stimuli in Experiment 1. 
different positions. The stimuli in these conditions were presented for $42 \mathrm{msec}$.

In the sparse irregular conditions, the stimuli consisted of $7 \times 3$ elements (mean extension, $13.98 \times 4.86 \mathrm{~cm}, 19.26^{\circ} \times 6.93^{\circ}$ ). The mean distance between the elements was $19.76 \mathrm{~mm}\left(2.83^{\circ}\right)$. The elements were placed with a positional jitter of \pm 20 pixels $( \pm 7.6 \mathrm{~mm}$, $\left.\pm 1.09^{\circ}\right)$. The target consisted of one single element. It could appear only in the middle row of the stimulus at all positions, except the two border positions. Thus, the target could appear at five different positions, with a maximal mean eccentricity of $6.5^{\circ}$. The stimuli were presented for $127 \mathrm{msec}$.

Design and Procedure. The combination of stimulus array (dense regular vs. sparse irregular) and target-context pair (open-targetclosed-context vs. closed-target-open-context) formed four conditions that were presented in separate blocks. In the dense regular conditions, a target was presented three times on each of the 13 possible positions, so that there were 39 positive trials (i.e., target-present trials) and 39 negative trials (i.e., target-absent trials) in a block, presented randomly. In the sparse regular conditions, a target was presented eight times on each of the 5 possible positions, so that a block consisted of 40 positive trails and 40 negative trails, presented randomly. Two blocks of each condition were presented.

Each stimulus presentation was preceded by a question mark displayed at the center of the screen, informing the participant that the computer was ready and that he or she could generate the first or next stimulus display. This was done by simultaneously pressing both mouse keys. The computer then replaced the question mark with a fixation point presented for $700 \mathrm{msec}$, followed by the stimulus, which was presented for $42 \mathrm{msec}$ (in the dense regular conditions) or for $127 \mathrm{msec}$ (in the sparse irregular conditions). ${ }^{2}$ The screen remained void until the participant responded by pressing either the left key (no target present) or the right key (target present). If the participant's response was correct, the keypress was followed by the question mark, indicating that a new trial could be initiated. When incorrect responses were made, the previous stimulus configuration was repeated for $1.5 \mathrm{sec}$ to provide feedback. This was followed by the question mark. Both reaction time and accuracy were recorded.

The participants were instructed to fixate on the fixation point as closely as possible, to respond as quickly as possible, and to make as few false alarms as possible. The last point was introduced to keep false alarms at a minimum, so that a participant would give a yes response only if he or she was relatively certain that the stimulus contained a target. The purpose of this instruction was to keep individual differences in criterion as low as possible. Since the hit rate varied as a function of the retinal eccentricity of the target and since the position of the target varied randomly within a block, the participants could define their criterion only in relation to the negative trials (trials without a target). As was proposed by M. Treisman and Watts (1966; see also Neyman \& Pearson, 1933), in such an experimental situation in which the signal strength varies within the experimental condition, it makes sense to use only the false alarm rate as an instruction for the participants to place their criterion.

Two sessions were administered on 2 successive days, each session lasting about $60 \mathrm{~min}$. In each session, either the two dense regular conditions or the two sparse irregular conditions were presented (two blocks of each condition, thus resulting in 156 trials per condition [dense regular] and 160 trials per condition [sparse irregular] for each participant). The sequence of the two conditions in each session and between the sessions was counterbalanced across participants. At the beginning of each session, a short practice sequence (dense regular condition, 26 trials, 1 target/position; sparse irregular condition, 20 trials, 2 targets/position) was performed, in which the relevant stimuli were presented for $141 \mathrm{msec}$. This practice sequence was followed by a (practice) block (as defined above) of each experimental condition delivered in the specific session (dense regular condition, 156 trials; sparse irregular condition, 160 trials) with the pre- sentation time of the experimental conditions (42 or $127 \mathrm{msec}$ ). The data of these practice blocks and of the practice sequence were not included in the data analysis.

\section{Results and Discussion}

Data summaries and analyses are based on the participants' percentages of correct detection of the target (hits). As was mentioned earlier, we have no information about the false alarm rate as a function of the different target positions. Therefore, we decided to compute $d^{\prime}$ values only for the data pooled over the different target positions. In addition, reaction times were analyzed to be sure that there were no speed-accuracy tradeoffs. Note that reaction times are based on rather different amounts of trials, because detection frequencies could vary considerably between conditions. Consequently, our main interest lies on the interpretation of the hit rate. For each participant, trials in which the reaction time exceeded the mean reaction time in this block by three standard deviations were not included in the analysis. This applies to data analyses in all the experiments.

Figure 2A (top row) presents the $d^{\prime}$ values for the four conditions. These data were submitted to a two-factor (stimulus array $\times$ target-context condition) repeated measures analysis of variance (ANOVA). The analysis showed a significant effect of stimulus array $[F(1,7)=$ $42.30, p<.001]$, no significant effect of target-context $[F(1,7)=1.90$, n.s. $]$, and a significantinteraction between stimulus and target-context $[F(1,7)=17.59, p<.005]$. As can be seen in Figure 2A, a closed target within open context elements was detected significantly more frequently than an open target within closed context elements for dense regular stimulus arrays $[F(1,7)=5.76, p<.05]$, whereas open targets within closed context elements were detected significantly more frequently than closed targets within open context elements for sparse irregular stimulus arrays $[F(1,7)=37.58, p<.0001]$. This interaction was mainly due to the effect of the spatial properties of the stimulus array in the open-target-closed-context condition. Detection rate in this condition was significantly higher for sparse irregular stimulus arrays than for dense regular array $[F(1,7)=92.74, p<.0001]$. On the other hand, the spatial properties of the stimulus array did not have any effect in the closed-target-open-context condition $(F=1)$.

Figures 2B-2C (top row) presents the percentage of hits as a function of eccentricity of the target. A two-factor (target-context condition $\times$ eccentricity) repeated measures ANOVA was performed separately for the dense regular and the sparse irregular conditions. The analysis for the dense regular stimulus showed a significant effect of eccentricity on detection performance $[F(12,84)=$ $16.57, p<.001]$ and a significant interaction between target-contextcondition and eccentricity $[F(12,84)=9.98$, $p<.001]$. Detection of an open target within closed-context elements dropped quickly with increasing eccentricity. On the other hand, performance for the closed-target-open-context condition was not best at the foveal area; rather, it in- 

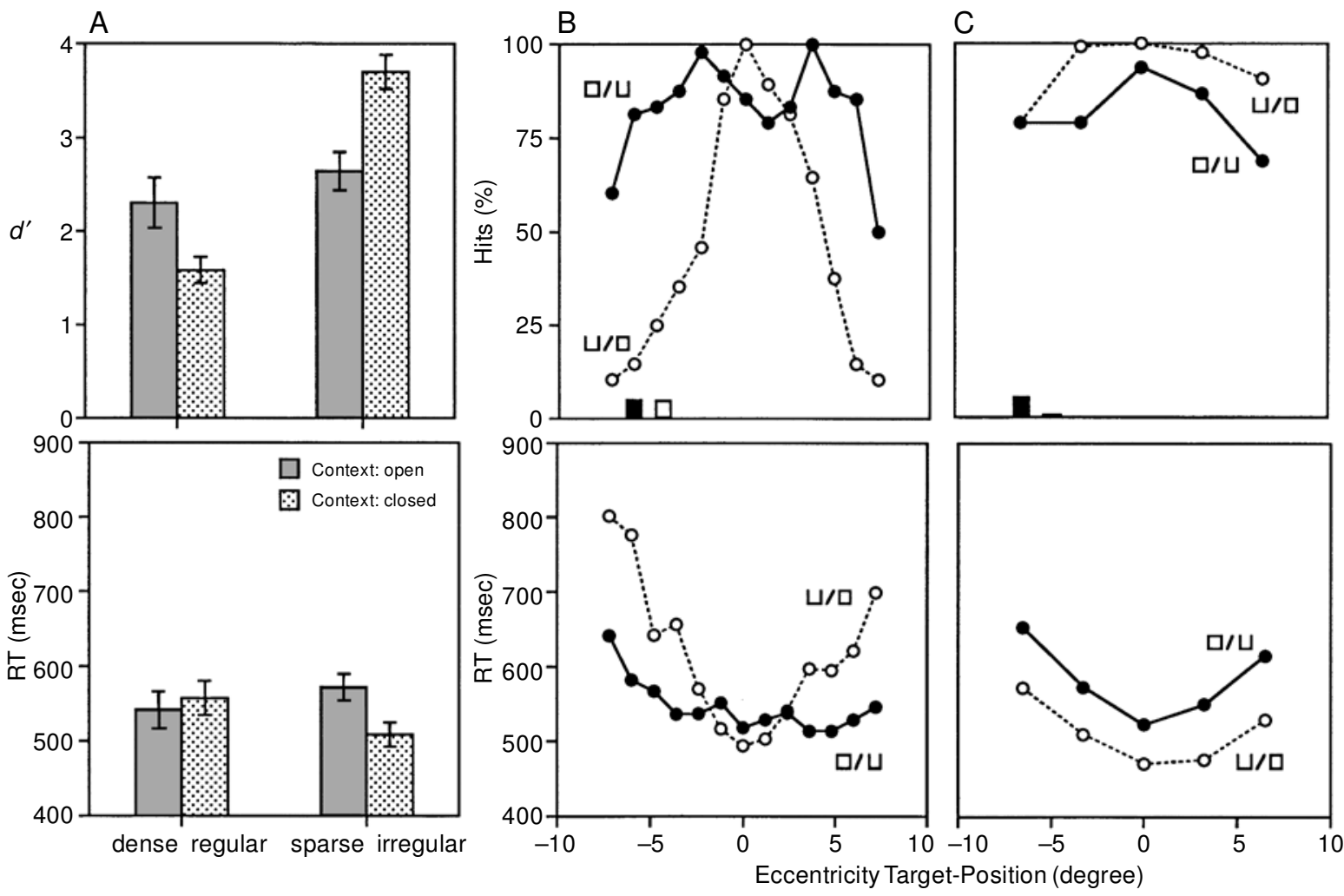

Figure 2. Results of Experiment 1. Top row: $d^{\prime}$ values (A) and mean hits per position of the target in the dense regular (B) and sparse irregular $(C)$ conditions. Blocks show false alarms. Bottom row: reaction time results.

creased with increasing eccentricity until it reached an eccentricity of about $3^{\circ}$, and at more eccentric positions, it started to decrease slowly. This pattern is similar to the CPD that was found with texture stimuli for which target and context lines differed in orientation (Gurnsey et al., 1996; Kehrer, 1987, 1989).

The analysis for the sparse irregular stimulus showed a significant effect of eccentricity $[F(4,28)=5.71, p<.01]$, but no significant interaction between target-context condition and eccentricity $[F(4,28)=2.14$, n.s.]. For both target-context conditions, performance decreased slowly with increased eccentricity.

The pattern of results is mirrored by the reaction time data (see Figure 2A, bottom row). Analysis of the data without consideration of the eccentricity of the target showed a significant effect of target-context $[F(1,7)=5.92, p<$ $.05]$, no significant effect of stimulus array $(\mathrm{F}<1)$, and a significant interaction between target-context and stimulus array $[F(1,7)=53.41, p<.001]$. This interaction is due to the significant difference between the two conditions in the sparse irregular array $[F(1,7)=47.06, p<$ $.001]$ and between the two open-target-closed context conditions $[F(1,7)=6.81, p<.05]$. When considering the eccentricity of the target (Figures 2B-2C, bottom row), the results are as follows. In the dense regular condition, eccentricity has a significant effect $[F(12,84)=$ $6.56, p<.001]$, and the interaction between the target- context condition and eccentricity is significant $[F(12,84)=$ $3.43, p<.001]$. In the sparse irregular condition, only eccentricity is significant $[F(4,28)=12.14, p<.001]$.

The results show clearly that the direction of the asymmetry between the two target-context conditions was reversed, depending on the spatial properties of the stimulus array and on retinal eccentricity of the target. Detection rate was higher for closed targets embedded in open elements than for open targets embedded in closed elements when the elements were arranged regularly with high density and were presented in the periphery. In contrast, open targets within the context of closed elements produced a higher detection rate than did closed targets within a context of open elements when presented in the foveal area, irrespective of the arrangement of the elements, and at any position when the arrangement was sparse and irregular. That is, when the target was presented foveally, an open target was detected more easily than a closed target. Performance with the open target decreased with increasing eccentricity of the target, presumably owing to a decrease in the resolution needed to perceive the open gap, but only with the dense regular displays was the performance decrease with eccentricity pronounced enough to reverse the asymmetry between the open and the closed targets.

In the present experiment, stimulus density, spatial regularity, and presentation time were correlated. The highly dense stimulus array was also highly regular and was pre- 
Sparse regular

\begin{tabular}{|lcccccc|}
\hline$\square$ & $\square$ & $\square$ & $\square$ & $\square$ & $\square$ & $\square$ \\
$\square$ & $u$ & $\square$ & $\square$ & $\square$ & $\square$ & $\square$ \\
$\square$ & $\square$ & $\square$ & $\square$ & $\square$ & $\square$ & $\square$ \\
$u$ & $u$ & $u$ & $u$ & $u$ & $u$ & $u$ \\
$u$ & $u$ & $u$ & $u$ & $u$ & $\square$ & $u$ \\
$u$ & $u$ & $u$ & $u$ & $u$ & $u$ & $u$
\end{tabular}

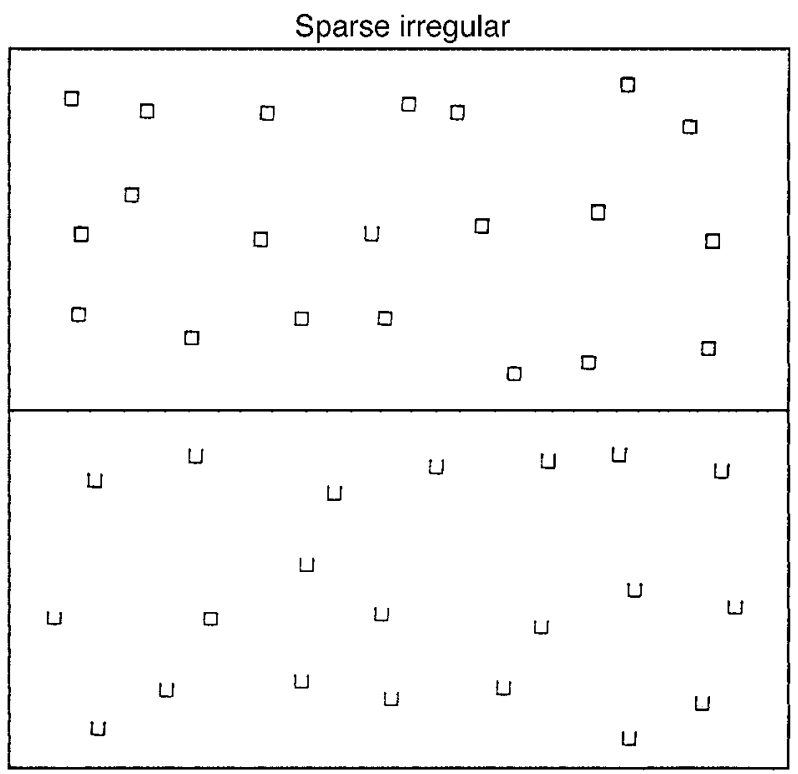

Figure 3. Stimuli in Experiment 2.

sented for $42 \mathrm{msec}$, whereas the sparse stimulus array both was highly irregular and was presented for $127 \mathrm{msec}$. Therefore, the results of this experiment do not allow us to assess the relative contribution of each of these factors to the reversal of asymmetry observed for the two stimulus conditions. In theory, each of these factors could have been critical for this reversal. Exposure duration may affect stimulus encoding, leading to different stimulus representations in the visual system and, thus, producing different asymmetries. Regularly arranged elements may produce a surface with certain properties that are not present in irregular stimulus arrays. Such global properties may be responsible for a reversal of the asymmetry. Similarly, differences in density also may produce different global properties that can be the cause for the switch in the direction of the asymmetry.

A further difference between the dense regular and the sparse irregular conditions is the number of elements that made up the target: In the dense conditions, the target patch consisted of three elements arranged vertically; in the sparse conditions, the target patch consisted of one single element. In principle, this could have caused the reversal in the asymmetry; one may claim, for example, that the task changed from a detection of a single target in the sparse irregular condition to a "texture" detection in the dense regular condition. In order to eliminate this confound between the spatial properties of the stimulus and the task, in the following experiments the target is always a single element.

In the following three experiments, we attempted to find out which of the mentioned factors (exposure duration, density, and regularity) are responsible for the asymmetry reversal. In Experiment 2, density was held constant at the low level; stimuli were presented for $42 \mathrm{msec}$ (as were the dense stimuli in Experiment 1) and were regularity varied. In Experiment 3, density was held constant at the high level and was regularity varied; in Experiment 4, regularity was held constant, and density was varied.

\section{EXPERIMENT 2 \\ Low Density: The Effect of Spatial Regularity}

In Experiment 2, we kept presentation time at $42 \mathrm{msec}$, held density constant at the low level, and varied the regularity of the stimulus array (see Figure 3 ). If the difference between the dense regular conditions and the sparse irregular conditions observed in Experiment 1 was due only to the difference in the exposure duration of the stimuli, the pattern of results for the low-density conditions in Experiment 2 would be similar to the one for the dense condition in Experiment 1, regardless of regularity. If, on the other hand, regularity were a critical factor, the pattern of results for the irregular condition in Experiment 2 would be similar to those for the irregular condition in Experiment 1, whereas that for the regular condition would be similar to the one for the dense regular conditions in Experiment 1.

\section{Method}

Participants. Eight participants (5 women and 3 men) were paid to participate in the experiment. They were 23-40 years old.

Apparatus. The apparatus was identical to that in Experiment 1.

Stimuli. The stimuli in the spatially irregular conditions were identical to the stimuli in the sparse irregular conditions in Experiment 1. In the spatially regular conditions, the stimuli were identical to the stimuli in the irregular conditions, with the exception that the elements were arranged completely regularly in the matrix (see Figure 3).

Design and Procedure. The design and procedure were identical to those of Experiment 1, with the exception that all the stimuli were presented only for $42 \mathrm{msec}$.

\section{Results and Discussion}

Figure 4A (top row) presents the $d^{\prime}$ values for the four conditions. A two-factor (regularity $\times$ target-context condition) repeated measures ANOVA showed a signifi- 
cant effect of regularity $[F(1,7)=20.26, p<.01]$, a significant effect of target-context condition $[F(1,7)=67.38$, $p<.001$ ], and no significant interaction between regularity and target-context condition $(F<1)$, indicating that the detection rate for an open target within closed context elements was significantly higher than the detection rate for a closed target within open context elements in both spatial arrangements; when the elements were arranged regularly, detection performance increased in both targetcontext conditions to the same extent.

Figures 4B and 4C (top row) present the percentages of hits as a function of eccentricity of the target. A two-way repeated measures ANOVA (target-context condition $X$ eccentricity) was conducted separately for the two regularity conditions. The analysis for the spatially regular condition showed a significant effect of eccentricity $[F(4,28)=$ $9.23, p<.01]$. The interaction between target-context condition and eccentricity was not significant $[F(4,28)=$ 0.10 , n.s.]. For both target-context conditions, performance decreased with increasing eccentricity. The analysis for the spatially irregular condition showed a significant effect of eccentricity $[F(4,28)=16.51, p<.01]$ and a significant interaction between target-context and eccentricity $[F(4,28)=2.77, p<.05]$. Performance in the closed-target-open-context condition decreased with ec- centricity faster than did performance in the open-targetclosed-context condition.

The pattern of results is mirrored by the reaction time data (see Figures $4 \mathrm{~A}-4 \mathrm{C}$, bottom row). There was a significant effect of target-context $[F(1,7)=19.73, p<$ $.005]$, a nearly significant effect of regularity $[F(1,7)=$ $5.35, p<.054]$, and a significant effect of eccentricity $[F(4,28)=18.16, p<.001]$. All interactions were not significant.

These results show that presentation time was not the critical factor in the asymmetry reversal found in Experiment 1: Under low density and spatial irregularity, an open target within closed context elements was detected better than a closed target within open context elements, even when the presentation time was only $42 \mathrm{msec}$. The level of performance in Experiment 2 was somewhat lower than that in Experiment 1, presumably owing to the shorter presentation time, but the direction of asymmetry was identical under both presentation times.

What effect did the manipulation of spatial regularity have? First of all, with spatially regular stimuli, the direction of asymmetry did not reverse, and the difference between the two target-context conditions did not diminish. This result may be taken as an indicator that spatial regularity is not the critical factor for the reversal of asymme-
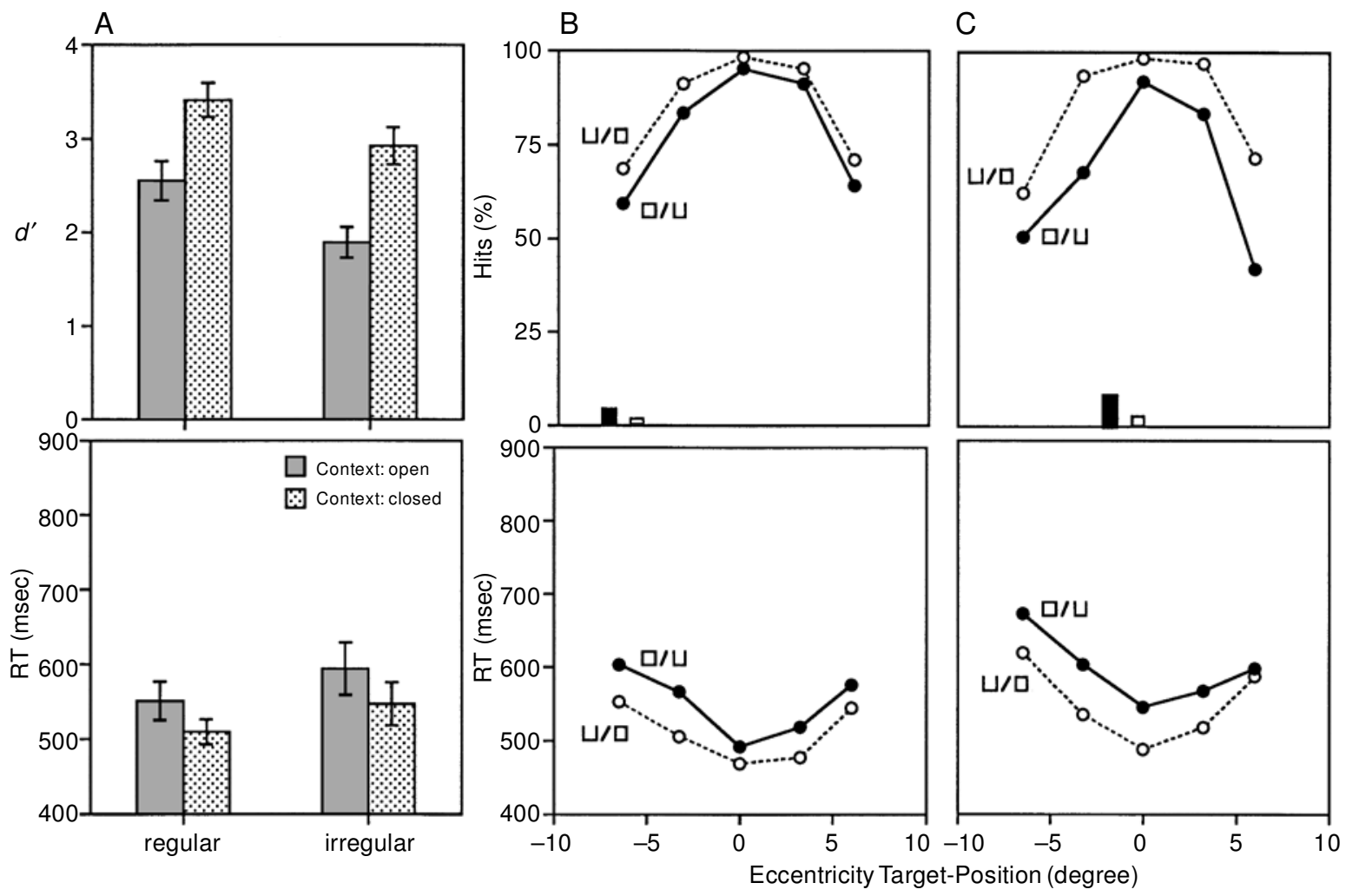

Figure 4. Results of Experiment 2. Top row: $d^{\prime}$ values (A) and mean hits per position of the target in the regular (B) and irregular $(C)$ conditions. Blocks show false alarms. Bottom row: reaction time results. 
try. Nevertheless, introducing spatial regularity improved performance slightly, a result that is in accordance with other findings (e.g., Gurnsey \& Browse, 1989; Kehrer, 1987; Nothdurft, 1990, 1991).

In sum, spatial regularity seems to have no differential effect on the two target-context conditions in stimuli with very sparsely arranged elements. Before we can reach any conclusions concerning the role of spatial regularity in the direction of detection asymmetry, we need to examine the effects of spatial regularity for dense stimulus arrays. The next experiment was designed to examine exactly this.

\section{EXPERIMENT 3 \\ High Density: The Effect of Spatial Regularity}

The results of Experiment 2 showed no differential effect of spatial regularity on detection asymmetry for very sparse stimulus displays. Experiment 3 was designed to find out whether regularity has a different effect on detection asymmetry for dense stimulus displays. Density was held constant at high level, presentation time was $42 \mathrm{msec}$, and regularity was varied.

In principle, it is not really possible to resolve this question, because a strong jitter cannot be realized in a dense arrangement without a positional overlap of the single elements. Nevertheless, we constructed a stimulus in which the elements were arranged at a somewhat lower density level than the one used in Experiment 1, but still higher than the one used in Experiment 2 so that it would be possible to insert a small jitter.

\section{Method}

Participants. Eight participants ( 6 women and 2 men) were paid to participate in the experiment. They were 21-35 years old.

Apparatus. The apparatus was identical to that in Experiment 1.

Stimuli. In the spatially regular conditions, the elements were regularly arranged in a $31 \times 7$ matrix $\left(151.24 \times 32.68 \mathrm{~mm}, 20.71^{\circ} \times\right.$ $\left.4.67^{\circ}\right)$. The distance between the single elements was $1.9 \mathrm{~mm}\left(0.27^{\circ}\right)$ in horizontal and vertical directions. The target could appear on the central row of the stimulus matrix on the central 23 positions, leaving each second position free. This resulted in 12 possible positions for a target, leaving the central position free. This was a result of the program used, which always centered all the possible target positions on the context matrix, and should be kept in mind when discussing the results. The target was presented three times on each possible position, resulting in 144 trials per condition per participant ( 12 positions $\times 3$ [per position] $\times 2$ [negative trials] $\times 2$ blocks). The stimuli in the spatially irregular conditions were identical to the stimuli in the spatially regular conditions described above, with the only exception that the elements were placed with a positional jitter of $\pm 0.38 \mathrm{~mm}\left( \pm 0.054^{\circ}\right.$; see Figure 5$)$. All other aspects of the stimuli were identical to those of Experiment 1.

Design and Procedure. The design and procedure were identical to those of Experiment 2.

\section{Results and Discussion}

Figure 6A (top row) presents the $d^{\prime}$ values for the four conditions. A two-factor (regularity $\times$ target-context condition) repeated measures ANOVA revealed no significant effect of regularity $[F(1,7)=2.65$, n.s. $]$, a significant effect of target-context condition $[F(1,7)=12.63, p<$ $.01]$, and a significant interaction between target-context condition and regularity $[F(1,7)=17.18, p<.01]$. Closed targets within open context elements produced a higher detection rate than did open targets within closed context elements in both regularity conditions $[F(1,7)=16.97$, $p<.01$, and $F(1,7)=7.40, p<.05$ ], for the spatially regular and the spatially irregular conditions, respectively]. Introducing a spatial jitter impaired performance for the closed-target-open-context condition $[F(1,7)=22.28$, $p<.01$ ], but not for the open-target-closed-context condition $(F<1)$.

Figures 6B and 6C (top row) presents the percentage of hits as a function of eccentricity of the target. A two-factor (eccentricity $\times$ target-context condition) repeated measures ANOVA was performed separately for the two spatial regularity conditions. The analysis for the spatially regular condition showed a significant effect of eccentricity $[F(11,77)=37.72, p<.001]$ and a significant interaction between target-context condition and eccentricity $[F(11,77)=9.55, p<.001]$. Detection performance for the open-target-closed-context condition dropped quickly with increased eccentricity, whereas the performance for the closed-target-open-context condition decreased slowly with increased eccentricity. Similar results were observed for spatially irregular stimulus arrays: There was a significant effect of eccentricity $[F(11,77)=45.42, p<.001]$ and a significant interaction between target-context condition and eccentricity $[F(11,77)=4.55, p<.001]$.

regular

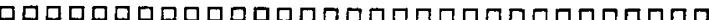
ㅁำ

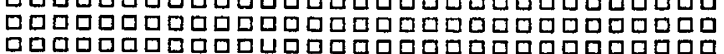

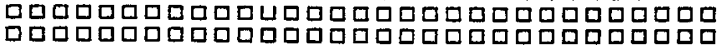


ด

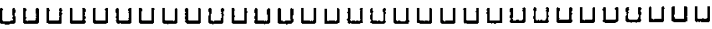

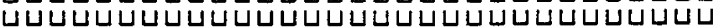



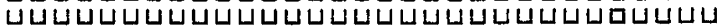

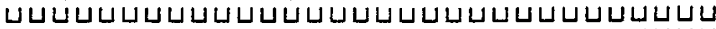
பபபபபபபபபபபபபபபபபபபபபபபபபบபபபபப

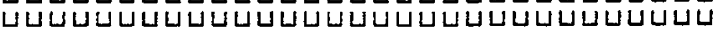

irregular

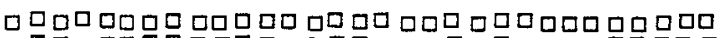

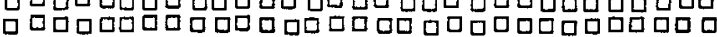
ด व ㅁำㅁำ ㅁำ ด هو

பபபப பபபப பபபபபப பபபப பீப பப பபபபபபபப பப பபபபப பப் பப பபபபபபபபப பபபபபபபப

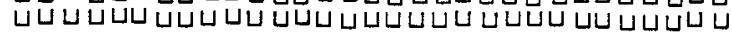
பபப பபபபபபபபபபபபபபபபபய பロ பப்பபபபப பபபபபபபபபபபபபபபபப பபபபபபபபப்பபபப

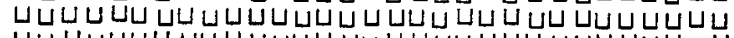

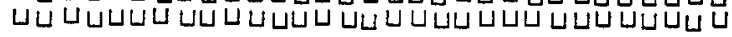

Figure 5. Stimuli in Experiment 3. 

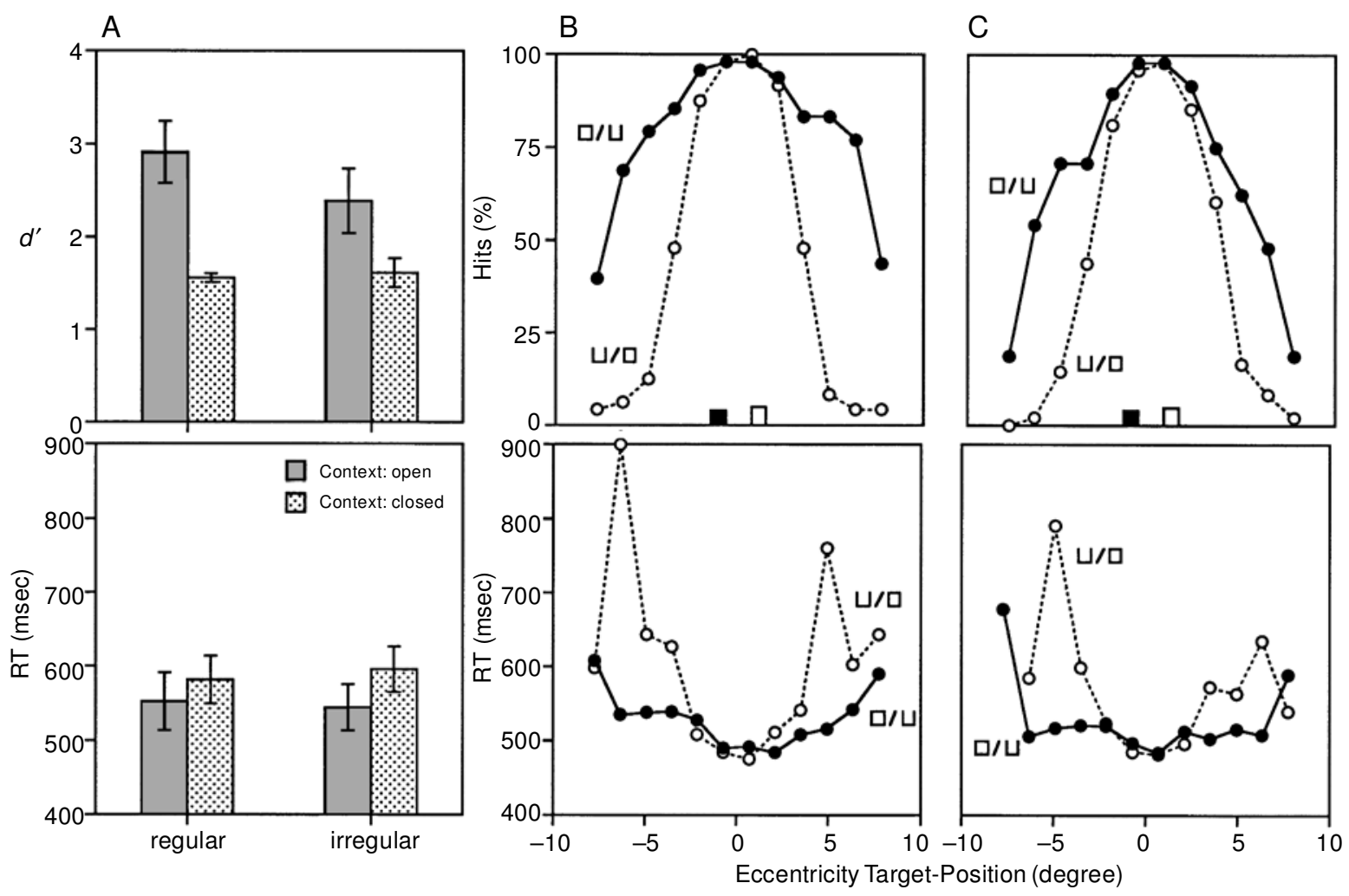

Figure 6. Results in Experiment 3. Top row: $d^{\prime}$ values (A) and mean hits per position of the target in the regular (B) and irregular $(C)$ conditions. Blocks show false alarms. Bottom row: reaction time results.

The ANOVA of the reaction time data (with the factors of regularity $\times$ target-context condition $\times$ eccentricity) showed the following significant results (see Figures 6A-6C, bottom row): eccentricity $[F(11,77)=12.46, p<.001]$, target-context condition $\times$ eccentricity $[F(11,77)=2.09$, $p<.05]$, and regularity $\times$ target-context condition $\times$ eccentricity $[F(11,77)=2.43, p<.05]$. Although in the open-target-closed-context condition, reaction times where slightly longer than in the other condition, thus mirroring the asymmetry in the percentage of hits data, this difference was not significant. The significant interaction between the three factors suggests that, as a function of regularity, the difference between the two target-context conditions takes a different course. A closer look at the data reveals that in the open-target-closed-context condition, at some (peripheral) target positions, the reaction time is strongly prolonged, and this variation is more pronounced in the regular condition than in the irregular condition. Since the number of hits at these positions is near zero, we refrain from a further discussion of these reaction time variations.

The results of Experiment 3 indicate that spatial regularity had an effect on the detection asymmetry: In the spatial irregularity condition, the magnitude of the asymmetry was reduced. This reduction was due to a performance decrement in the closed-target-open-context condition as a function of spatial irregularity. Note, however, that in both spatial conditions (with and without jitter), the asymmetry was rather pronounced. It was observed particularly in peripheral areas. In the foveal area, performance was at ceiling in both conditions.

The CPD for the condition with open context elements, observed in Experiment 1, was not visible in this experiment. Two aspects of the data may be responsible for this failure to replicate it. First, performance was at ceiling in the foveal area. Second, in this experiment, the CPD had a reduced chance to occur, because no target was presented in the central position of the stimulus. One may assume that two other differences between Experiment 1 and Experiment 3 could be responsible for the lack of a CPD. First, it is possible that rather high density is required to produce CPD, and the density in Experiment 3 was somewhat lower than that in Experiment 1. Second, it is possible that a target consisting of more than one element is necessary (a texture target) to produce a CPD, and the target in Experiment 3 was a single element rather than a $3 \times 1$ array, as in Experiment 1 . As for the density difference, Kehrer (1989) has shown that decreasing the density makes the CPD more pronounced. As for the difference in the target, Meinecke $(1995,1997)$ and Meinecke and Donk (2002) have shown that a CPD can be observed also with a target consisting of one single element.

Taken together, the results of Experiments 2 and 3 suggest that spatial regularity may have some effect on the mag- 
Medium high density

ロロロロロロロロロロロロロロロロロロロロロロロ



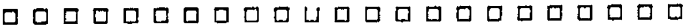

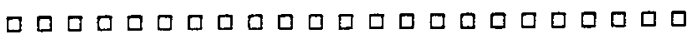

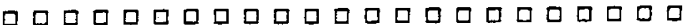

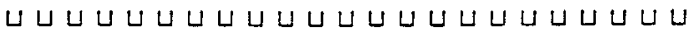

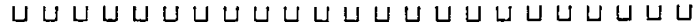

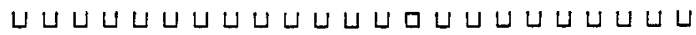

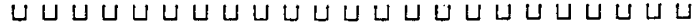
பபபபபபபபபபபபபபแபபபபபபபப

Medium low density

\begin{tabular}{|c|c|c|c|c|c|c|c|c|c|c|c|c|c|c|}
\hline ם & ם & 口 & 口 & 口 & $\square$ & 口 & 口 & $\square$ & ם & 口 & $\square$ & ㅁ & $\square$ & ם \\
\hline$\square$ & 口 & $\square$ & $\square$ & ㅁ & $\square$ & 口 & $\square$ & 口 & $\square$ & ם & $\square$ & 口 & $\square$ & 口 \\
\hline 口 & $\square$ & 口 & ם & $\cup$ & $\square$ & $\square$ & ㅁ & 口 & 口 & $\square$ & $\square$ & $\square$ & $\square$ & $\square$ \\
\hline ם & $\square$ & $\square$ & ט & $\square$ & 口 & 口 & $\square$ & ם & 口 & $\square$ & 口 & $\square$ & $\square$ & 口 \\
\hline 口 & $\square$ & 口 & 口 & 口 & $\square$ & ם & $\square$ & 口 & 口 & $\square$ & 口 & 口 & ㅁ & 口 \\
\hline$\sqcup$ & $U$ & $\cup$ & $\sqcup$ & $\sqcup$ & $\sqcup$ & $U$ & $\cup$ & 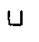 & $u$ & $\sqcup$ & $\sqcup$ & $u$ & $\cup$ & $山$ \\
\hline$\sqcup$ & $\sqcup$ & $\sqcup$ & $\mathrm{U}$ & $\cup$ & $\cup$ & $\sqcup$ & $\sqcup$ & $u$ & $u$ & $u$ & $\cup$ & $\sqcup$ & $u$ & $U$ \\
\hline$\sqcup$ & $\sqcup$ & $\sqcup$ & $\cup$ & $\sqcup$ & $\cup$ & $\sqcup$ & $\sqcup$ & $\sqcup$ & $\sqcup$ & $\cup$ & $山$ & 口 & U & $\sqcup$ \\
\hline$\sqcup$ & $U$ & $\sqcup$ & $\sqcup$ & $山$ & $\sqcup$ & $u$ & $u$ & $\cup$ & $\sqcup$ & $\cup$ & $\sqcup$ & $\sqcup$ & $山$ & $\sqcup$ \\
\hline$\sqcup$ & $\sqcup$ & $\sqcup$ & $u$ & $\omega$ & $\omega$ & $\cup$ & $u$ & $\sqcup$ & u & $\sqcup$ & $u$ & $\sqcup$ & $\sqcup$ & $\sqcup$ \\
\hline
\end{tabular}

Figure 7. Stimuli in Experiment 4.

nitude of detection asymmetry, but in neither case was the effect strong enough to produce a qualitative differencenamely, a reversal in the asymmetry. Rather, the results show clearly that the detection rate of open targets within a closed context was higher than the detection rate of closed target within open context elements for low-density stimulus arrays (Experiment 2), whereas the opposite holds for high-density stimulus arrays (Experiment 3 ).

In the next experiment, we further examine the role of the density of the stimulus array for the reversal of the detection asymmetry.

\section{EXPERIMENT 4 Low Versus High Density}

The results of Experiments 1-3 are seen to suggest that spatial density plays an important role in the direction of detection asymmetry for open and closed elements. In Experiment 4 , we held regularity constant and varied density, using two levels of density, (medium) high and (medium) low, to get more information about the effect of density on detection asymmetry.

\section{Method}

Participants. Eight participants (5 women and 3 men) were paid to participate in the experiment. They were 21-40 years old.
Apparatus. The apparatus was identical to the one in Experiment 1.

Stimuli. In the low-density conditions (see Figure 7), the elements were regularly arranged in a $15 \times 5$ matrix $(136.04 \times 41.04 \mathrm{~mm}$, $\left.18.78^{\circ} \times 5.86^{\circ}\right)$. The distance between the single elements was $6.46 \mathrm{~mm}\left(0.93^{\circ}\right)$ in the horizontal and vertical directions. The target could appear on the central row of the stimulus matrix, with equal probability on the central 11 positions. On each possible position, the target was presented three times, resulting in 132 trials per condition per participant (11 positions $\times 3$ [per position] $\times 2$ [negative trials] $\times 2$ blocks). In the high-density conditions, the elements were regularly arranged in a $23 \times 5$ matrix $\left(145.16 \times 28.88 \mathrm{~mm}, 19.95^{\circ} \times\right.$ $\left.4.13^{\circ}\right)$. The distance between the single elements was $3.42 \mathrm{~mm}$ $\left(0.49^{\circ}\right)$ in the horizontal and vertical directions. The target could appear on the central row of the stimulus matrix, with equal probability on the central 17 positions. On each possible position, the target was presented twice (136 trials per condition). All other aspects of the stimuli were identical to those in Experiment 1. Note that the low density in this experiment was somewhat higher than the low density in Experiments 1 and 2 and that the high density was somewhat lower than the high density in Experiments 1 and 3. We chose these two intermediate densities to fill the gap between the already tested densities in Experiments 1, 2, and 3.

Design and Procedure. The design and procedure were identical to those in Experiment 1, with the exception that all the stimuli were presented for $42 \mathrm{msec}$.

\section{Results and Discussion}

Figure 8A (top row) presents the $d^{\prime}$ values for the four conditions. A two-factor repeated measures ANOVA (density $\times$ target-context condition) showed no significant main effect of density or of target-context condition $[F<1$ and $F(1,7)=1.18$, n.s., respectively]. The interaction between density and target-context condition was significant $[F(1,7)=9.42, p<.02]$. Closed targets within open context elements were detected significantly more frequently than open targets within closed context elements for the high-density stimulus array $[F(1,7)=5.86, p<$ $.05]$, but there was no significant difference between the two target-context conditions for the low-density stimulus array $(F<1)$.

Figures $8 \mathrm{~B}$ and $8 \mathrm{C}$ (top row) present the percentage of hits as a function of eccentricity of the target. A two-way repeated measures ANOVA (eccentricity $\times$ target-context condition) was conducted separately for the low- and the high-density conditions. The analysis for the low density condition showed a significant effect of eccentricity $[F(10,70)=12.32, p<.001]$ and a significant interaction between target-context condition and eccentricity $[F(10,70)=1.98, p<.05]$. Detection performance for the open-target-closed-contextcondition tended to drop with eccentricity faster than did detection performance for the closed-target-open-contextcondition, and there was some hint of a CPD for the latter. The analysis for the high-density condition also showed a significant effect of eccentricity $[F(16,112)=19.40, p<.001]$ and a significant interaction between target-context condition and eccentricity $[F(16,112)=3.95, p<.001]$. There was some hint of a CPD for the closed-target-open-context condition, and detection performance for the open-target-closed-context condition dropped with eccentricity faster than did de- 

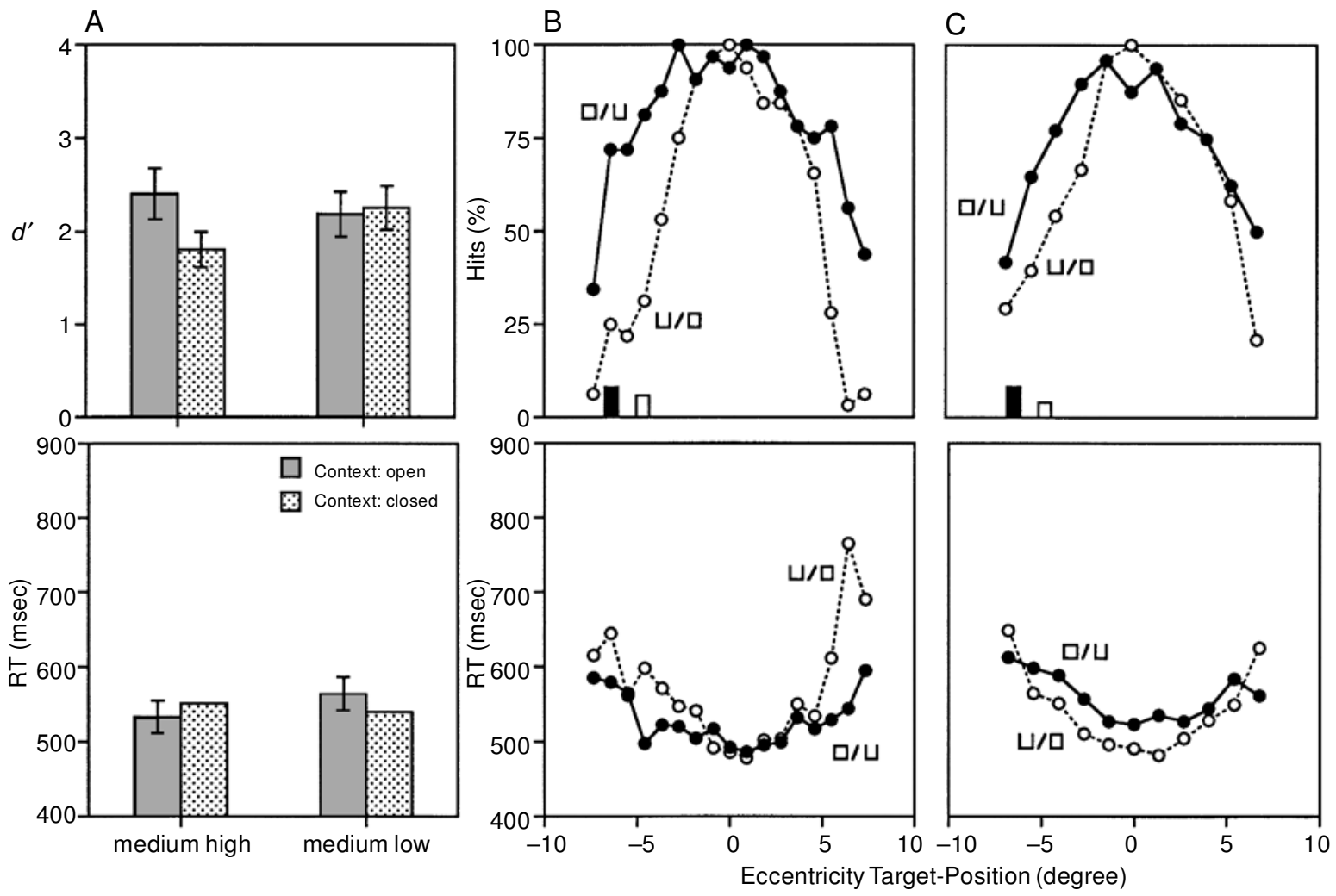

Figure 8. Results in Experiment 4. Top row: $d^{\prime}$ values (A) and mean hits per position of the target in the medium high (B) and medium low (C) density conditions. Blocks show false alarms. Bottom row: Reaction time results.

tection performance for the closed-target-open-context condition.

Reaction time results are shown in Figures $8 \mathrm{~A}-8 \mathrm{C}$, bottom row. Neither the density factor $(F<1)$ nor the targetcontext condition factor $[F(1,7)=5.07$, n.s.] was significant. The interaction between these two factors was significant $[F(1,7)=6.73, p<.05]$. This significant interaction was due to a significant difference in reaction times between the two target-context conditions in the lowdensity condition $[F(1,7)=9.92, p<.02]$. An analysis of the eccentricity data for each density condition separately revealed only a significant effect of position in both density conditions $[F(16,112)=7.07, p<.001$, for high density; $F(10,70)=4.69, p<.001$, for low density].

These results suggest that density may be a critical factor in the asymmetry reversal. The asymmetry that was observed for the high-density condition in Experiments 1 and 3-namely, that a closed target within an open context was detected more frequently than an open target within a closed context - was replicated in the present experiment for the high-density condition, but it disappeared when the density was reduced (i.e., in the low-density condition) and even was reversed in the reaction time data. Granted that the density used in the present experiment for the low-density condition was higher than the density for the low-density conditionin Experiments 1 and 2, one could assume that re- ducing the density in the low-density condition even more in the present experiment would result in a reversal in detection asymmetry in the direction observed in Experiment 1 in the detection frequency data, too.

The difference between the direction of the asymmetry in foveal and in peripheral areas that was observed very clearly in Experiment 1 for the high-density condition was not as clearly observed in the present experiment. Presumably, performance in the present experiment was at ceiling in the foveal area, thus hiding an advantage of the opentarget-closed-context condition over the closed-targetopen-context condition.

\section{GENERAL DISCUSSION}

An asymmetry in detection performance has been reported in the literature when stimuli are constructed with open and closed target and context elements (e.g., A. M. Treisman \& Souther, 1985; Williams, 1992): It is easier to find an open element embedded in closed elements than to find a closed element embedded in open elements. In our experiments, this finding was replicated, but a reversal of the direction of this asymmetry was also observed.

Several factors were varied in our experiments, including the density of the elements, the spatial jitter of the elements, and the retinal eccentricity of the target position. 


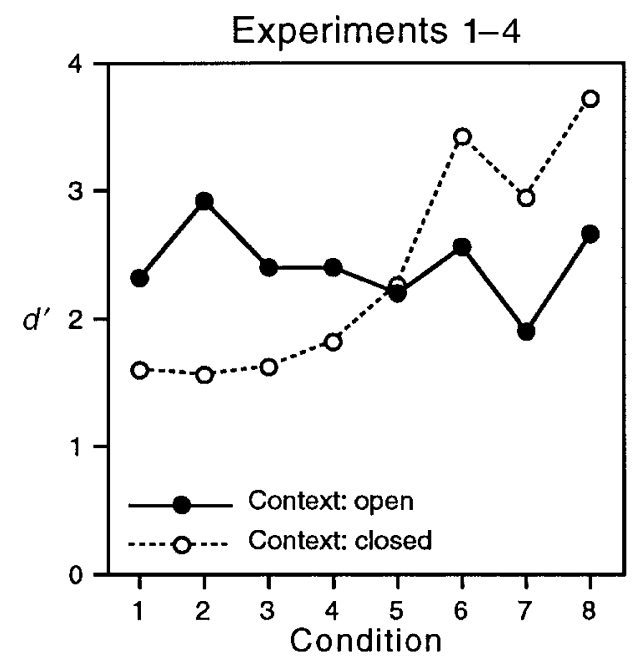

\begin{tabular}{|l|l|l|l|l|l|l|l|l||}
\hline No. of Condition & 1 & 2 & 3 & 4 & 5 & 6 & 7 & 8 \\
\hline \hline Experiment & 1 & 3 & 3 & 4 & 4 & 2 & 2 & 1 \\
\hline Spacing (pixel) & 1 & 3 & 3 & 7 & 15 & 50 & 50 & 50 \\
\hline Jitter (pixel) & 0 & 0 & 1 & 0 & 0 & 0 & 20 & 20 \\
\hline Display duration (msec) & 42 & 42 & 42 & 42 & 42 & 42 & 42 & 127 \\
\hline
\end{tabular}

Figure 9. Results of Experiments 1-4.

In order to discuss the results of all the experiments together, we ordered the different conditions of the experiments according to the following logic (see Figure 9): In a first step, the conditions were arranged according to their degree of density; if two conditions had the same density (as, e.g., in Experiment 3), the factor of jitter determined their order; if two conditions were identical with respect to density and jitter (Experiments 1 and 2), they were ordered according to their presentation duration.

Although the results stem from different experiments, a clear pattern seems to emerge, which can be described as follows. (1) There was no systematic effect of density on the detection of a closed target within open context elements. Only introducing a spatial irregularity (jitter) led to a performance decrement (Condition 2 vs. Condition 3 ; Condition 6 vs. Condition 7). (2) There was a systematic effect of density on the detection of an open target within closed context elements. Detection was very efficient with sparse stimulus arrays, significantly better than the detection of closed target within open context elements, thus producing the asymmetry reported in the literature. Performance decreased as the density of the stimulus increased, so that with dense stimulus arrays it was significantly poorer than the detection of closed target within open context elements, producing a reversal in the direction of the asymmetry. Introducing spatial irregularity (jitter) impaired performance in the sparse arrangement (Condition 6 vs. Condition 7). Prolonging the display duration increased performance in both conditions.
The pattern of results regarding eccentricity can be summarized as follows. (1) For both target-context conditions, performance decreased with increasing eccentricity. But for the open-target-closed-context condition, this performance decrease with increased eccentricity occurred more strongly and more quickly as the density of the stimulus array increased, whereas for the closed-target-opencontext condition, detection performance as a function of eccentricity was hardly affected by stimulus density. (2) For both target-context conditions, performance in the foveal area was at ceiling in most situations, possibly hiding existing differences between the target-context conditions. In the most dense, regular arrangement (Experiment 1), a CPD coupled with a peripheral performance increase was observed for the closed-target-open-context condition. A hint of a similar CPD effect was also observed in Experiment 4 .

These results suggest that an explanation of detection asymmetry that focuses only on stimulus properties (either of the target, as in the more-the-better hypothesis, or of the context, as in the deviation hypothesis) cannot account for detection asymmetries in different stimulus and presentation conditions. Rather, we suggest that the grain of analysis (e.g., Meinecke, 1989), which varies as a function of stimulus density and of retinal eccentricity, interacts with stimulus content to affect detection performance. That is, depending on the grain of analysis, single elements and their properties or larger units of the stimulus array and more global properties are more readily available. 
Consequently, the effects of increased density and eccentricity would be detrimental for a detection that is based on single-element properties, but not for a detection that is based on more global properties. Properties of single elements, such as, for example, terminators (or a gap), are more likely to be perceived in the foveal area and with a sparse element arrangement than in the peripheral areas and with a dense element arrangement. It is known that increased density reduces the perception of the properties of single elements. This was demonstrated, for example, by Bouma (1970), and the effect is known as lateralmasking, lateral inhibition or the crowding effect (Strasburger, Harvey, \& Rentschler, 1991). It is also known that this effect increases with increasing eccentricity (e.g., De Valois $\&$ De Valois, 1988). Therefore, a task that involves finding a property of a single element is expected to be performed very efficiently in the foveal area and with sparse arrays but to be hindered when density and eccentricity increase. This is exactly the pattern of performance that we found for the detection of an open target within closed context elements, suggesting that the perception of detailed information-of properties of the single elements (e.g., terminators) - is critical for this detection task.

On the other hand, the perception of properties that are computed over larger units of the stimulus array (e.g., homogeneity/heterogeneity) is less likely to be hindered by dense element arrangement, and increased eccentricity does not necessarily have a detrimental effect; rather, it may even be beneficial. Indeed, several studies of texture discrimination show that increasing the density of texture stimuli can improve detection performance (e.g., Sagi \& Julesz, 1987; Nothdurft, 1985). Therefore, a detection task that involves more global properties is expected not to be affected by increased density of the stimulus array, and the effect of eccentricity is expected to be relatively small. Such a pattern of performance was observed for the detection of a closed target within open context elements: Detection with a sparse stimulus array was not as efficient as that for an open target within closed context elements, but increasing density did not hinder performance, and there was a rather slow performance decrease with increased eccentricity (and even some benefit, to a degree, from increased eccentricity; see Experiment 1). These findings suggest that detection performance in this case was based on a property that emerged from the stimulus array as a whole (e.g., heterogeneity/homogeneity).

Now, if a certain detection task can be performed on the basis of properties that are best supported by the available stimulus and presentation conditions, this task will show an advantage over a task for which these conditions are not as favorable. This can result not only in detection asymmetry, but also in a reversal in the direction of the asymmetry, as was observed in Experiments 1 and 4. Thus, for example, if the detection of an open target within closed context elements can be best performed by detecting terminators or a gap, whereas the detection of a closed target within open context elements can be best performed by detecting homogeneity/heterogeneity, the former will show an advantage relative to the latter under conditions that are favorable for detecting a unique property of a single element (i.e., foveal area, sparse array) but will show a disadvantage under conditions that are not favorable for such a detection and at the same time are not harmful (and may be even beneficial) for the perception of homogeneity/ heterogeneity (i.e., peripheral area, dense array). This would result in a reversal in detection asymmetry.

The idea that different properties may mediate target detection, depending on the available stimulus and presentation conditions, is also supported by the observation made by Wolfe (1992) that properties that support parallel visual search need not be the same as the ones that support effortless texture segmentation, and vice versa. Wolfe attributed this observation to the different tasks (search for a target vs. segmentation) and suggested that when, for the same stimuli, texture segmentation is easy but search is difficult, texture segmentation may be based on a global property. It should be noted, however, that the distinction between search tasks and texture tasks in terms of the task is not so clear. Although typical search and typical texture segmentation may differ so that the former requires a search for a predefined target, whereas the latter requires that one texture be segmented from another (e.g., Julesz, 1981), many of the studies that used tasks that are considered "texture tasks" required, in fact, detection of a target (either a single element or a group of elements; e.g., Beck, 1982; Meinecke \& Kehrer, 1994; Nothdurft, 1985), much as in a search task. Actually, a more robust difference between "texture tasks" and search tasks has to do with the spatial properties of the stimulus: The typical display for texture segmentation is dense and regular, whereas the typical display for search task is sparse and irregular. In our experiments, we used the same stimuli with the same detection task under different stimulus conditions that varied from dense and regular to sparse and irregular and showed that detection performance varies as a function of the properties of the display, suggesting that, presumably, different properties mediate detection performance under the different stimulus conditions.

We suggest, then, that a detection task that can utilize the properties that are available with a given grain of analysis would show an advantage over a task for which the given grain of analysis is not as favorable, resulting in detection asymmetry in one direction or another. It is important to note that our suggestion implies that detection asymmetry is a result of an interaction between target-context properties and stimulus and presentation conditions. It does not specify, however, which specific stimulus properties are responsible for good or poor detection performance.

Our suggestion also implies that there is a grain of truth in either of the hypotheses mentioned in the introduction: the more-is-better hypothesis and the deviation hypothesis. The former, which emphasizes the specific properties of the target, is more compatible with fine-grained units of analysis that allow the detection of unique element prop- 
erties. The latter, which emphasizes detection of discontinuity, is more compatible with more coarse-grained units of analysis that allow the detection of discontinuity or irregularity in the stimulus array. That is, the detection of an open target within closed context elements is accomplished by a search for unique elements, as is implied by the more-is-better hypothesis. The detection of a closed target within open context elements is accomplished by the detection of irregularity in the stimulus array. Indeed, the only exception to the rather constant performance of this task across different densities was observed when irregularity was introduced into the display. Several studies have also shown that spatial irregularity can impair detection performance (e.g., Gurnsey \& Browse, 1989; Kehrer, 1987; Nothdurft, 1990, 1991).

The direction of the asymmetry is often used as a diagnostic tool for determining visual primitives (e.g., A. M. Treisman \& Gormican, 1988), but the validity of this diagnostic tool has been questioned (e.g., Carrasco et al., 1998). Our finding that the direction of the asymmetry can be reversed as a function of stimulus density and eccentricity casts further doubt on the validity of this diagnostic tool. Yet, the finding that variations in spatial density and in eccentricity have differential effects on detection performance can be informative as to the actual size of grain of analysis that is responsible for detection performance.

\section{REFERENCES}

Appelle, S. (1972). Perception and discrimination as a function of stimulus orientation: The oblique effect in man and animals. Psychological Bulletin, 78, 266-278.

Bacon, W. F., \& Egeth, H. E. (1991). Local processes in preattentive feature detection. Journal of Experimental Psychology: Human Perception \& Performance, 17, 77-90.

Beck, J. (1973). Similarity of curves. Perceptual \& Motor Skills, 36, 1331-1341.

BECK, J. (1982). Textural segmentation. In J. Beck (Ed.), Organizationand representation in perception (pp. 285-317), Hillsdale, NJ: Erlbaum.

Bouma, H. (1970). Interaction effects in parafoveal letter recognition. Nature, 226, 177-178.

Carrasco, M., \& Frieder, K. S. (1997). Cortical magnification neutralizes the eccentricity effect in visual search. Vision Research, 37, 63-82.

Carrasco, M., McLean, T. L., Katz, S. M., \& Frieder, K. S. (1998). Feature asymmetries in visual search: Effects of display duration, target eccentricity, orientation and spatial frequency. Vision Research, 38, 347-374.

De Valois, R. L., \& De Valois, K. K. (1988). Spatial vision. New York: Oxford University Press.

Foster, D. H., \& WARD, P. A. (1991). Horizontal-vertical filters in early vision predict anomalous line-orientation identification frequencies. Proceedings of the Royal Society of London: Series B, 243, 83-86.

GuRnSEY, R, \& BROWSE, R. A. (1987). Micropattern properties and presentation conditions influencing visual texture discrimination. Perception \& Psychophysics, 41, 239-252.

Gurnsey, R., \& Browse, R. A. (1989). Asymmetries in visual texture discrimination. Spatial Vision, 4, 31-44.

Gurnsey, R., Pearson, P., \& Day, D. (1996). Texture segmentation along the horizontal meridian: Nonmonotonicchanges in performance with eccentricity. Journal of Experimental Psychology: Human Perception \& Performance, 22, 738-757.

Joffe, K. M., \& Scialfa, C. T. (1995). Texture segmentation as a function of eccentricity, spatial frequency and target size. Spatial Vision, 9, 325-342.

JuLESZ, B. (1981). Figure and ground perception in briefly presented isodipole textures. In M. Kubovy \& J. R. Pomerantz (Eds.), Perceptual organization (pp. 27-54). Hillsdale, NJ: Erlbaum.

Julesz, B., \& BERGEN, J. R. (1983). Textons, the fundamental elements in preattentive vision and perception of textures. Bell System Technical Journal, 62, 1619-1645.

Kehrer, L. (1987). Perceptual segregation and retinal position. Spatial Vision, 2, 247-261.

KeHRER, L. (1989). Central performance drop on perceptual segregation tasks. Spatial Vision, 4, 45-62.

Kimchi, R., \& Navon, D. (2000). Relative judgment seems to be the key: Revisiting the Beck effect. Journal of Experimental Psychology: Human Perception \& Performance, 26, 789-805.

Meinecke, C. (1989). Retinal eccentricity and the detection of targets. Psychological Research, 51, 107-116.

MeInECKe, C. (1995). How early is early vision? Familiarity effects in texture segmentation and their relation to retinal eccentricity. Perception, 24, 131-132.

MeInecke, C. (1997). Texture segmentation and the familiarity effect [Abstract]. Perception, 26, 111.

Meinecke, C., \& Donk, M. (2002). Detection performance in pop-out tasks: Nonmonotonic changes with display size and eccentricity. Perception, 31, 531-602.

Meinecke, C., \& Kehrer, L. (1994). Peripheral and foveal segmentation of angle textures. Perception \& Psychophysics, 56, 326-334.

Neyman, J., \& Pearson, E. S. (1933). On the problem of the most efficient tests of statistical hypotheses. Philosophical Transactions of the Royal Society of London: Series A, 231, 289-337.

Nothdurft, H. C. (1985). Sensitivity for structure gradient in texture discrimination tasks. Vision Research, 25, 1957-1968.

NothduRFT, H. C. (1990). Texton segregation by associated differences in global and local luminance distribution. Proceedings of the Royal Society of London: Series, B, 239, 295-320.

NothduRfT, H. C. (1991). Different effects from spatial frequency masking in texture segregation and texton detection tasks. Vision Research, 31, 299-320.

Poirier, F. J. A., \& Gurnsey, R. (1998). The effects of eccentricity and spatial frequency on the orientation discrimination asymmetry. Spatial Vision, 11, 349-366.

Rubenstein, B. S., \& SAGi, D. (1996). Preattentive texture segmentation: The role of line terminations, size, and filter wavelength. Perception \& Psychophysics, 58, 489-509.

SAGI, D., \& JulEsz, B. (1987). Short-range limitation on detection of feature differences. Spatial Vision, 2, 39-49.

Strasburger, H., Harvey, L. O., Jr., \& Rentschler, I. (1991). Contrast thresholds for identification of numeric characters in direct and eccentric view. Perception \& Psychophysics, 49, 495-508.

Treisman, A. M., \& Gormican, S. (1988). Feature analysis in early vision: Evidence from search asymmetries. Psychological Review, 95, 15-48.

Treisman, A. M., \& Souther, J. (1985). Search asymmetry: A diagnostic for preattentive processing of separable features. Journal of Experimental Psychology: General, 114, 285-310.

Treisman, M., \& Watts, T. R. (1966). Relation between signal detectability theory and the traditional procedures for measuring sensory thresholds: Estimating $d^{\prime}$ from results given by the method of constant stimuli. Psychological Bulletin, 66, 438-454.

Wang, Q., Cavanagh, P., \& Green, M. (1994). Familiarity and pop-out in visual search. Perception \& Psychophysics, 56, 495-500.

Williams, D. W. (1992). Cooperative parallel processing in depth, motion and texture perception. In J. Brannan (Ed.), Applications of parallel processing in vision (pp. 167-225). Amsterdam: Elsevier.

Wolfe, J. M. (1992). "Effortless" texture segmentation and "parallel" visual search are not the same thing. Vision Research, 32, 757-763.

Yeshurun, Y., \& CARRAsco, M. (1998). Attention improves or impairs visual performance by enhancing spatial resolution. Nature, 396, 72-75.

\section{NOTES}

1. We chose open and closed squares instead of the more commonly used circles (e.g., Rubenstein \& Sagi, 1996; A. M. Treisman \& Souther, 1985 ) because detection performance was found to be especially difficult with round elements as stimuli (Meinecke, 1989). 
2. This difference in presentation time between the dense regular and the sparse irregular conditions has historical reasons. We started this study with pilot experiments with dense regular texture-like stimuli, presented for $42 \mathrm{msec}$, and could not replicate the direction of asymmetry reported in the literature in visual search studies. We modified the stimuli and presentation conditions to resemble visual search conditions more closely. In visual search tasks, stimuli are usually presented without time limitation. But since we wanted to measure performance as a function of eccentricity, we reduced presentation time to under $150 \mathrm{msec}$, to $127 \mathrm{msec}$. This is usually short enough to prevent eye movements from occurring during stimulus presentation.

(Manuscript received April 25, 2000; revision accepted for publication October 21, 2001.) 